\title{
Il dopo delle famiglie: disagi, quasi certezze e speranze
}

\author{
Michele Corsi
}

\begin{abstract}
L'articolo si compone di tre paragrafi, con riferimento alle famiglie dopo questa pandemia da coronavirus. Un dopo che è anche un durante. Perché non sappiamo nemmeno quanto durerà. E, cioè, i disagi, le quasi certezze e le speranze. Partendo da un assunto: ci pare davvero le che reali situazioni familiari siano pressoché sconosciute a chi ci governa. Nondimeno con un romanticismo che ci sembra inopportuno e fuori luogo. I disagi: un eccesso di competenze che di fatto non si posseggono a fronte di un virus per lo più sconosciuto; le decisioni dello Stato e le maggiori situazioni di povertà, non soltanto economiche, nel nostro Paese; la distanza del potere nei riguardi del popolo; e la didattica a distanza per il suo interfacciarsi con le reali possibilità delle famiglie italiane. Le quasi certezze: un aumento di separazioni e divorzi nel prossimo futuro e il peggioramento di dinamiche familiari già compromesse. Le speranze: che lo Stato, la Chiesa e anche la pedagogia lavorino maggiormente e più proficuamente in futuro a favore delle culture della stabilità e del progetto. Delle famiglie come delle persone. Passando per i giovani e gli attuali adulti.
\end{abstract}

Parole chiave: coronavirus, famiglie, pedagogia, stabilità, progetto.

\begin{abstract}
The paper focuses on the situation of families after coronavirus pandemic, and it is divided in three sections. An "after", which is also a "now", because we do not even know how long it will last. We, therefore, need to discuss the discomforts, the almost certainties and the hopes, starting from a consideration: it seems that the situations families are living these days are virtually unknown to those governing us. In addition, what it possibly even worse, the latter behave using a romanticism, which seems inappropriate and out of place. The discomforts: an excess of competences no one really possess to fight an almost unknown virus; the decisions taken by the Government and the major situations of poverty - and not only the economic one - of our Country; the distance between Power and People; and distance learning, in light of the real possibilities of Italian families. The almost certainties: an increase in separations and divorces in the near future and a worsening of already compromised family dynamics. The hops: that the State, the
\end{abstract}

${ }^{1}$ Professore ordinario di Pedagogia generale e sociale presso l'Università degli Studi di Macerata e Direttore del Dipartimento di Scienze della formazione, dei Beni culturali e del Turismo dello stesso Ateneo. 
Church, and Pedagogy as well, work more and more fruitfully in the near future towards project and stability cultures for the families and the people, from young ones to adults.

Keywords: coronavirus, families, pedagogy, stability, project.

\section{Introduzione}

Voglio essere breve. Anche perché, in un periodo di incertezze come questo, penso che siamo tutti sufficientemente stanchi (ed è un eufemismo) di sentir pontificare da troppi di sicurezze che non possiedono, di scenari che sono a dir poco altamente ipotetici e di direttrici di marcia legate più al sogno, se non all'incubo, che alla realtà. Oportet tacere. $\mathrm{O}$ almeno i più, per quanto utile e possibile.

Quanto stiamo vivendo, infatti, è un tempo in progress sconosciuto e sospeso. Che ignoriamo quasi del tutto. Ma da trascorrere assieme. Attraversandolo con la bussola dei marinai, sperando di non andarci a perdere o incagliare da qualche parte (come il Titanic) e dunque sprofondare. Con la maggiore lucidità possibile. Con la mente sgombra da pregiudizi. Che sono, invece, tanti in questo momento. E con un cuore altrettanto fermo.

In attesa del vaccino, alla cui collaborazione internazionale già gli USA e la Cina hanno dichiarato di non voler partecipare ${ }^{2}$.

\section{I disagi}

Partiamo dalle famiglie. E dalle famiglie in Italia: queste sconosciute. Com'è proprio del fascicolo di questa rivista. Con questo articolo che è in progress, al pari di questo presente. Specie per quando uscirà, e sarà disponibile ai lettori. Con molte situazioni che potrebbero cambiare o persino peggiorare nel frattempo; e con commenti e giudizi, i miei, che potrebbero, quindi, essere pure diversi. Totalmente e parzialmente. $\mathrm{Ma}$ che fotografa, per intanto, questi ultimi due mesi di marzo e aprile 2020.

$\mathrm{E}$ inizio dai disagi.

${ }^{2} \mathrm{Si}$ fa per completezza presente che l'articolo in oggetto è stato redatto e chiuso prima della realizzazione e della somministrazione, in Italia e nel mondo, dei vaccini anti SARS-CoV-2, N.d.R. 
Uno l'ho già rappresentato: la "vendita" eccessiva e fuori luogo di competenze che non si hanno e non si possono avere. O comunque per ciò che si manifesta, si dice ecc. Questo virus non lo conosciamo. Nessuno l'ha mai visto, né incontrato. E va dunque studiato con tutta l'umiltà della scienza. Di quella vera, non parolaia e neppure paranoica. Come oggi, invece, accade troppo spesso. Che il virus, ad esempio, tornerà in autunno. Anzi no, a gennaio. Che gli fa bene il caldo. Oppure no? Che non riapriamo le scuole per la sicurezza degli alunni. Ma i bambini e i giovani non sono quelli meno soggetti al contagio? Allora per gli insegnanti? Cogli anziani, sullo sfondo, che dovrebbero essere, al contrario, tutti più o meno rincitrulliti e quindi desiderosi di ammalarsi appena mettono il naso fuori casa. Ma che, quando passò la pensione a quota 100, li si descrisse come dei giovanotti. E che sinora sono andati a fare la spesa, ritirare la pensione ecc. Per giunta nella fase 1 . Mascherine sì e mascherine no. Real politik: perché ne abbiamo poche? Mascherine, poi, a 0,50 centesimi l'una. Sempre che sia davvero questo il prezzo calmierato. E con due euro al giorno, dunque, per una famiglia "media" di quattro persone.

Come la mettiamo con i probabili 10 milioni di prossimi poveri nel nostro Paese? $\mathrm{O}$ col reddito universale che si dovrebbe aggirare intorno ai 500-600 euro mensili? A riceverlo, poi, puntualmente. Cui andrebbero aggiunti anche i guanti. Sarà una banale influenza? Come ha affermato l'OMS tra gennaio e febbraio di quest'anno, elogiando addirittura la Cina per la sua strategia verso il coronavirus. E secondo il parere, in passato, pure di alcuni componenti dell'ISS. Che tuttora dissentono da altri virologi. Infine no: una pandemia. Penso che posso fermarmi qui. Invocando per l'attuale isolamento, auto-isolamento ecc.: resistenza e resilienza. E nemmeno scarse o di poco conto, queste ultime.

Il secondo disagio. Abbiamo fatto anche un breve corso d'inglese in queste settimane, alla televisione. Tanti termini, tanti anglicismi sbattuti in faccia a un popolo disorientato e spaventato che voleva capire. $\mathrm{O}$, meglio, che avrebbe voluto capire, per orientarsi. Mi viene in mente una pagina dei Promessi sposi: il parlare difficile per ribadire la distanza del potere. E, oltre a tutto questo English, a volte nondimeno un linguaggio da iniziati. Per pochi eletti. E non certamente per il popolo: una categoria da non dismettere mai. Oppure come quando il cappellaio matto in Alice nel paese delle meraviglie sostenne che il termine "gloria" poteva assumere tanti significati a seconda di chi comandava.

Il terzo. Il romanticismo insulso e d'accatto di queste settimane. Noiosissimo e persino pruriginoso a tratti. Avremmo preferito piuttosto, 
ovunque e comunque, una comunicazione più sobria pure per rispetto di quanto stavamo vivendo. Con interviste e propagande anche di sapore idilliaco. Favolistico. C'era una volta, e tutti vissero felici e contenti. Ci mancavano Filemone e Bauci, i fidanzatini alla Peynet, i "baci Perugina" ecc. ed eravamo a posto. Specie dopo aver gridato, in questi ultimi decenni, alla morte della famiglia. Di una famiglia, poi, costantemente al singolare, forse perché era di questo modello in particolare che ci si voleva sbarazzare: ritenuto un retaggio borghese e pure anti-democratico. $\mathrm{E}$, cioè, della famiglia stabile poggiata su un istituto abbracciato e convenuto. In specie sul sacramento del matrimonio. Ma forse anche quello su base civile, celebrato in Comune, a poter dare fastidio. E adesso tutti assieme in casa. Che bello! Per giunta in appartamenti di $40 \mathrm{mq}$, la più parte. Come ha notato di recente Chiara Saraceno. Con famiglie spesso di quattro persone. Quando non di più. E, tornando alla prima, con due figli pure di età diversa impegnati nella didattica a distanza. Sempre a disporre di computer, tablet ecc., a sufficienza e per ciascuno. E con le connessioni a funzionare. Che invece cadevano di continuo. E magari con un genitore almeno a lavorare anche in smart working.

Mi taccio. Come se tutti, infatti - ma è opportuno che mi ripeta -, abitassimo in grandi appartamenti o peraltro bastevoli, avessimo giardini e terrazze ecc. Dimenticavo: ci sono i balconi (nondimeno per chi li ha). E là, tutti a cantare. Uno sull'altro. Perché il contagio in famiglia non si realizza (vero?), ma solo quando usciamo o facciamo footing oppure andiamo al parco. Ma qui giustamente distanziati, con guanti e mascherine. Mentre i contagi intra-familiari sembrano essere, al contrario, il $25 \%$ del totale, il $4 \%$ quello sui luoghi di lavoro e il $44 \%$ nelle case di riposo (fonte: Protezione civile).

Il quarto, e il più grave. L'ignoranza - ribadisco - in capo alle nostre famiglie attuali. Tutte rigorosamente (come nella réclame della pasta De Cecco) al plurale. Esistono in Italia 15 differenti modelli di organizzazioni familiari. Tra cui le famiglie separate, divorziate e ricostituite. Come se di queste ultime i nostri governanti e parlamentari non fossero esperti. Eppure è la loro condizione di vita, pure in pochi vertici. Esiste anche un esercito di due milioni di minorenni, in un Paese tra l'altro non incline alla procreazione, che vive per lo più con le mamme. E coi padri lontani. Quanto li avranno potuto incontrare in questi due mesi? Specie a risiedere, costoro, pure in un altro comune o fuori regione. Mentre anche il mito della genitorialità è stato propagandato.

$\mathrm{E}$, infine, i single. Un'altra schiera sterminata, particolarmente tra i 35 enni e i 40enni. Ma questi, forse, più attrezzati informaticamente. 
Insieme, ma a distanza. E potrei continuare all'infinto. Con numerosi altri esempi e rimandi. Ma su un aspetto non posso non soffermarmi. Quello delle coppie già spaiate, che erano in attesa di andare dal giudice per separarsi e divorziare. E ora no, insieme. In case sovente minuscole, quando non sotto-scale.

Per ritornare in qualche modo pure alla didattica a distanza, perché collegata alla dinamica odierna delle famiglie. Che ha funzionato magnificamente, ha detto la Ministra Azzolina. Scuole e famiglie che hanno collaborato ottimamente tra loro e tutte tecnologiche. Allora, perché è stato stanziato un fondo di 85 milioni per dotare le scuole di computer? Non avevano già tutto? E i genitori, tutti laureati, in una nazione come la nostra che è il fanalino di coda o quasi dell'istruzione superiore in Europa? E i genitori, che hanno continuato a lavorare, quando e come avranno aiutato i figli nei compiti? Di notte? Quando i bambini e i ragazzi dormivano o volevano vedere la tv? E i genitori immigrati che sanno a malapena l'italiano? E, ancora, didattica a distanza o da distante? Tanto da celebrare addirittura, come esempio luminoso cui tornare al presente, la trasmissione Non è mai troppo tardi del maestro Manzi. Di 60 anni fa, o sbaglio? Alla faccia del progresso, delle tecnologie avanzate in Italia e così via. In buona sostanza, se molto o abbastanza ha funzionato da noi, è per il solito miracolo dell'auto-organizzazione degli italiani.

Davvero: le famiglie, queste sconosciute da parte di chi ci governa. O, meglio, ci dovrebbe governare. Con saggezza, competenza e lungimiranza.

Consentitemi due sole altre battute.

La prima, tornando alle famiglie: siamo consapevoli che a volte o spesso, durante l'adolescenza in particolare, i rapporti fra i genitori e i figli sono di pessima grana? Invece, adesso, due mesi in posizione ultraravvicinata?

E quindi le violenze familiari (Ulivieri, 2015). Tanto che ora si è iniziato a dare un certo numero di telefono per soccorrere in specie le donne che si trovano in queste condizioni.

\section{Le quasi certezze}

E una in particolare. Aumenteranno le separazioni, almeno nel breve periodo. Oltre a quelle già in programma. Dopo due mesi di contatto gomito a gomito tra persone minimamente abituate a stare assieme, questa overdose di prossimità significherà un "Basta!" grande come l'Empire 
State Building. Tra soggetti, fra l'altro, abituati a vivere, negli ultimi decenni, più nei gruppi secondari, e cioè nei luoghi di lavoro ecc., che non in quelli primari, e, cioè, in casa (Speltini, Palmonari, 2007).

E anche le richieste di psicoterapia cresceranno. Da parte di quelle famiglie che vorrebbero almeno provarsi nella durata. Per le crisi di coppia da rimettere in piedi per quanto possibile, come per i rapporti sgangherati e peggiorati fra genitori e figli. A potersele permettere, però. Perché quelle costano. Così d'avverarsi, una volta di più, la profezia di Fornari di qualche tempo fa: la nevrosi, malattia dei poveri, curata ai ricchi. E sempre che il welfare sociale da noi riparta. Con bilanci in passato, e maggiormente sarà in futuro, ridotti al lumicino. Con consultori familiari pressoché azzerati o medicalizzati in gran parte d'Italia e coi servizi di salute mentale privi di personale adeguato ecc.

$\mathrm{Ma}$, in aggiunta, non si parlava di crisi dell'educazione fino a ieri? La CEI non ha emanato al riguardo, nel 2010, un documento: Educare alla vita buona del vangelo?

Affermando tutti, ma sino all's marzo 2020 (che però qui non è la festa della donna), che non avevamo più adulti in Italia (o almeno solo all'anagrafe), che i figli e i giovani erano abbandonati a loro stessi, che confondevano l'amore col fare l'amore, che il precocismo sessuale era la loro risposta malsana e sbagliata al bisogno d'intimità che avevano, e non di poco.

Ora tutto superato? Siamo diventati, all'improvviso, un popolo di individui educati (eccetto quel 5\% - irrisorio! - che non ha rispettato le regole, tra cui i "poveri di spirito" e forse pure qualche claustrofobico), di famiglie felici, stabili e ricche di solidarietà reciproca, come di ottime relazioni inter-generazionali. Un Paese "per bene". Tutti di memoria corta, in Italia. Anche la Chiesa. Per non parlare del Governo, della classe politica e della tv di Stato. Ci avete fatto caso che, in questi due mesi, sono pressoché scomparsi pure gli omosessuali dalla ribalta televisiva? Non parlo di qualche nostro intrattenitore. E, figuratevi, mi sono tutti simpatici. Ma non un accenno a costoro. Ai problemi che possono avere incontrato anche loro in questo periodo: questi nostri invisibili (Savarese, 2015; Stramaglia, 2015), in tanti, di questa nostra strana postmodernità. E nessun riferimento nemmeno alle unioni civili o alle prime, sparute, famiglie omosessuali. Niente di niente. Eppure sino a poco tempo fa non c'era una fiction, da noi, che non annoverasse una situazione del genere nella sua narrazione. 


\section{Le speranze}

In specie se la pedagogia si sveglia. Se inizia a fare discorsi scientifici e moralmente prospettici. Ancorché dovutamente articolati. A farsi davvero carico della pedagogia delle famiglie. Di tutte, nessuna esclusa. Non tralasciando alcuna delle tipologie familiari esistenti. E, con essa, dei nostri concittadini. O almeno dei più. Per il loro bene e quello dei loro figli.

Due culture sono in crisi in Italia e da tempo: quelle della stabilità e del progetto.

Separazioni e divorzi oltre il $40 \%$ in molte regioni italiane e il $77 \%$ dei fallimenti per quanto riguarda i matrimoni misti. Che sono già, questi ultimi, il $12 \%$ del nostro Paese (fonte: ISTAT, media degli ultimi cinque anni). E immaginarsi domani, quando queste relazioni in futuro cresceranno per i tanti immigrati di seconda e terza generazione che ci sono in Italia.

Sono un tifoso della curva di Gauss o della distribuzione normale dei fenomeni. Va tutto bene se il segno della differenza viene contenuto nel 17,6\% periodico di questa rappresentazione. Separazioni e divorzi, cioè, entro questo flusso. E così ogni distanziamento (e oggi ci sta bene questo termine) da una norma "di bene". O la stabilità è un male? La normalità: a bene intenderla però!, è una cosa preistorica e fuori moda? La normalità della decisione libera, autonoma e responsabile. E pure qui con riferimento a tutte le situazioni di vita, nessuna esclusa. Vivere da parte dei figli con genitori separati e divorziati, in più case e colla valigia pronta per i fine settimana a intervallo tra la mamma e il papà, è un valore da perseguire? Ma cosa abbiamo fatto, scritto e soprattutto operato noi pedagogisti per ridurre tutti questi dati? E lo Stato quando mai si è preoccupato della formazione alla vita di coppia e familiare? E la Chiesa cattolica? Con due errori fondamentali che quest'ultima ha commesso. Il primo: quello di confondere la coppia con la famiglia. Di scrivere e agire maggiormente sulla famiglia che non sulla coppia, perché più adusi, sacerdoti e vescovi, alla prima che non alla seconda. Perché cresciuti in famiglia; ma senza esperienza concreta, e spesso faticosa nelle scelte e nelle decisioni, della vita di coppia. Oppure limitandosi per quest'ultima, sovente sovrapponendo indebitamente e senza mediazioni il magistero con la pastorale, alla memoria continua, incessante e martellante, e spesso improvvida, del rispetto del sesto comandamento, a "predicare" i metodi naturali, a stigmatizzare separazioni e divorzi, come se di taluni matrimoni, regalati in fretta e senza avvedutezza, fosse stata solo spettatrice. Come se il matrimonio e la convivenza fossero 
unicamente sessualità, letti disfatti ecc., e non una quotidianità estremamente complessa.

Anche nella somministrazione dei sacramenti la Chiesa ha pasticciato e non poco. Elargendoli "a tempo" e di fatto raramente interconnettendoli fra loro, con cammini in dovuta prosecuzione. Il battesimo alla nascita, e quindi pausa; il sacramento dell'eucarestia e il lungo balzo in avanti a quello della cresima. Con linguaggi spesso antiquati e metodi non rinnovati. E dunque il lunghissimo silenzio, sovente fino a trent' anni e oltre, sino al matrimonio e alla sua preparazione: limitato per giunta a una minoranza. Con incontri o catechesi per già iniziati. Quando invece molti "fidanzati" sono pressoché analfabeti di ritorno alla fede. O dandoli per scontati tutti vergini o giù di lì.

Un altro fenomeno in crescita, nel mondo e pure in Italia, è quello omosessuale. Sondaggi recenti ci dicono, ad esempio, che un giovane inglese su due non si definisce eterosessuale al $100 \%$ e nemmeno se ne preoccupa. Con lo stesso comportamento a riguardare anche un terzo della popolazione statunitense come di altre nazioni europee, compreso il nostro Paese (Corsi, 2016). Specie in capo alla componente femminile. Pure qui c'è da farsi carico del problema, che tale è. Tornando i padri a fare i padri e le madri le madri. Oggi c'è molta confusione di ruoli, di stili e di approcci. Se è vero, com'è vero, che la nostra personalità è il frutto delle molte identificazioni che si sono succedute nel tempo. E a chi, allora, questi nostri fratelli e queste nostre sorelle avranno guardato, per scegliere liberamente, responsabilmente e autonomamente (Corsi, 2003)? Perché ognuno di noi è l'erede di ciò che è stato, ha avuto e ha vissuto. Ed è nondimeno la "conseguenza" dinamica e interattiva del copione trans-generazionale della propria famiglia (Hellinger, Ten Hövel, 2005, trad. it. 2013).

La cultura contemporanea è anche contrassegnata, come ho scritto poc'anzi, dalla caduta del progetto. $\mathrm{O}$, a voler essere benevoli, da una sua diffusa eclisse. Quel progetto della singola persona come di un gruppo o delle differenti micro e macro comunità al pari della società nella sua generalità, che nasce dal passato di un'esistenza individuale, oppure collettiva, insiste sul presente per proiettarsi nel futuro, e lo occupa. Ma che oggi, in una sorta di metafora agostiniana perversa o alla maniera del modello, reinterpretato contemporaneamente, dello «slancio vitale» del francese Bergson (1907, trad. it. 2012, passim), viene messo da parte. Per essere diventato, tutto, un eterno presente. Rifiutato e negletto il passato, col taglio di molteplici radici. E con un futuro altrettanto posto in non cale, non concepito e non accolto, perché fumoso, aereo, vago, 
indistinto, confuso, incerto e improbabile (e chi più ne ha, più ne metta). $\mathrm{E}$ che non a caso questa pandemia potrebbe, magari pure a torto, aver rinforzato. Se non si imparano piuttosto, da questa lezione, un diverso uso del tempo e una differente visione della vita.

Archiviando comunque pressoché definitivamente questa traiettoria temporale, il progetto come gettatezza nell'avvenire conseguentemente viene meno. E tutto finisce col racchiudersi in un qui e ora dilatato all'infinito dalla mente e dalle proiezioni umane. Innanzitutto negli adulti e nei tardo-adulti, come accade e non raramente, e quindi, alla luce di questi "modelli" difettosi o mancanti, negli adolescenti e nei giovani adulti con le loro scelte di quasi perpetuo bricolage affettivo-sentimentale e con una decisione di singletudine protratta (Corsi, 2011). E senza dimenticare che, almeno fino a ieri, erano in molti, moltissimi o troppi, gli adulti (all'anagrafe) che rincorrevano l'eterna giovinezza con restyling estetici continui, che negavano gli anni che avanzavano, che lasciavano cadere rapporti consolidati e coevi per rincorrere un "amore" ben più giovane, nell'assurda follia di riportare indietro il tempo, per contagio. $\mathrm{O}$ a saltare aste impossibili, a 70 anni: tutti teenager, sbarazzini, e di più.

Il coronavirus, la malattia e la morte hanno fatto fuori: per noi definitivamente o quasi, questa illusione deformante. Non siamo quello che vogliamo apparire, siamo quello che siamo. Gli anni e la carta d'identità non si truccano. Il dentro batte inesorabilmente il fuori. Ugualmente, ci sono gli anziani nel nostro Paese: il 27\% della popolazione.

Allora, rimbocchiamoci tutti le maniche. C'è tanto da fare, e per i pedagogisti in primis, al fine di scrivere un futuro diverso per i nostri figli, per i nostri giovani, per tutte le nostre coppie: nessuna esclusa, e per le nostre famiglie in avvenire. Personalmente lo vorrei tanto, e di più lo spero!

\section{Riferimenti bibliografici}

Bergson H. (1907): L'evoluzione creatrice. Trad. it. Milano: Biblioteca Universale Rizzoli.

Corsi M. (2003): Il coraggio di educare. Il valore della testimonianza. Milano: Vita e Pensiero.

Corsi M. (2011): Famiglia e famiglie: dalla singletudine alla solidarietà. In L. Martiniello (a cura di): L'infanzia in una stagione di crisi. Napoli: Guida, pp. 117-133.

Corsi M. (2016): La bottega dei genitori. Di tutto e di più sui nostri figli. Milano: FrancoAngeli. 
Hellinger B., Ten Hövel G. (2005): Riconoscere ciò che è. La forza rivelatrice delle costellazioni familiari. Trad. it. Milano: Feltrinelli, 2013.

Savarese E. (2015): Lettera di un omosessuale alla Chiesa di Roma. Roma: Edizioni e/o.

Speltini G., Palmonari A. (2007): I gruppi sociali. Bologna: il Mulino.

Stramaglia M. (2015): L'invisibilità delle coppie omosessuali. L'esigenza etica di diritti civili. In M. Tomarchio, S. Ulivieri (a cura di): Pedagogia militante. Diritti, culture, territori. Roma: Anicia, pp. 261-268.

Ulivieri S. (2015, a cura di): Corpi violati. Condizionamenti educativi e violenze di genere. Milano: FrancoAngeli. 